\title{
Fibroblast Growth Factor-9 Activates c-Kit Progenitor Cells and Enhances Angiogenesis in the Infarcted Diabetic Heart
}

\author{
Dinender Singla and Jing Wang \\ Biomolecular Science Center, Burnett School of Biomedical Sciences, College of Medicine, University of Central Florida, \\ Orlando, FL 32816, USA \\ Correspondence should be addressed to Dinender Singla; dsingla@ucf.edu
}

Received 27 April 2015; Accepted 9 August 2015

Academic Editor: Massimo Collino

Copyright (C) 2016 D. Singla and J. Wang. This is an open access article distributed under the Creative Commons Attribution License, which permits unrestricted use, distribution, and reproduction in any medium, provided the original work is properly cited.

\begin{abstract}
We hypothesized that fibroblast growth factor-9 (FGF-9) would enhance angiogenesis via activating c-kit positive stem cells in the infarcted nondiabetic and diabetic heart. In brief, animals were divided into three groups: Sham, MI, and MI+FGF-9. Two weeks following MI or sham surgery, our data suggest that treatment with FGF-9 significantly diminished vascular apoptosis compared to the MI group in both C57BL/6 and db/db mice $(p<0.05)$. Additionally, the number of c-kit ${ }^{+v e} / \mathrm{SM} \alpha$-actin ${ }^{+\mathrm{ve}}$ cells and c-kit ${ }^{+\mathrm{ve}} / \mathrm{CD} 31^{+\mathrm{ve}}$ cells were greatly enhanced in the MI+FGF-9 groups relative to the MI suggesting FGF-9 enhances c-Kit cell activation and their differentiation into vascular smooth muscle cells and endothelial cells, respectively $(p<0.05)$. Histology shows that the total number of vessels were quantified for all groups and our data suggest that the FGF- 9 treated groups had significantly more vessels than their MI counterparts $(p<0.05)$. Finally, echocardiographic data suggests a significant improvement in left ventricular output, as indicated by fractional shortening and ejection fraction in both nondiabetic and diabetic animals treated with FGF-9 $(p<0.05)$. Overall, our data suggests FGF-9 has the potential to attenuate vascular cell apoptosis, activate c-Kit progenitor cells, and enhance angiogenesis and neovascularization in $\mathrm{C} 57 \mathrm{BL} / 6$ and $\mathrm{db} / \mathrm{db}$ mice leading to improved cardiac function.
\end{abstract}

\section{Introduction}

Diabetes, characterized by dysregulated circulating blood glucose levels, is consequent to pancreatic beta cell destruction yielding little to no insulin production (type I, insulindependent diabetes mellitus, IDDM) or insulin resistance stemming from genetic predisposition, age, obesity, hypertension, and/or sedentary lifestyle habits (type II, noninsulin dependent diabetes mellitus, NIDDM). Myocardial infarction (MI), in the context of NIDDM patients, is much more prevalent compared to nondiabetic counterparts with associated increased risk of post-MI morbidity and mortality [1-3]. Consequent to MI in NIDDM patients, dynamic, complex, and adverse vascular and myocardial remodeling results in an attempt to rescue endogenous left ventricular structure and function. Such processes are characterized by (1) cell death via apoptosis and necrosis of cardiac cell types including cardiac myocytes, (2) fibroblast infiltration and scar formation, (3) hypertrophy, and (4) vascular cell death including vascular smooth muscles (VSM) and endothelial cell (EC) types [4-7]. Previous reports have suggested that abnormal myocardial angiogenesis in the setting of diabetes may be resultant of enhanced vascular cell type death and dysregulated angiogenic growth factors and cytokines such as vascular endothelial growth factor (VEGF) and angiopoietin1 (Ang-1) [8-10]. Gene therapy has been attempted, with use of various factors including Ang-1, in diabetic animal models to promote appropriate vascular maturation and development [8-10]. Although significant improvement in impaired angiogenesis was noted, optimal gene therapy has yet to be identified in NIDDM patients and animal models and remains a major challenge.

Fibroblast growth factors (FGFs) comprise a large family of polypeptide growth factors that contribute to a host 
of biological functions including embryonic development, tissue morphogenesis, and physiological homeostasis [11, 12]. In particular, FGF-9, like other family members, has been shown to mediate variegated cellular processes including neuronal cell growth and development, midgestational myocardial proliferation and coronary neovasculogenesis, testicular embryogenesis, hair follicle neogenesis, inner ear morphogenesis, and joint development [13-19]. Recently, data has been published suggesting conditional transgenic FGF-9 expression in the post-MI heart enhanced microvessel density and left ventricular hypertrophy, reduced interstitial fibrosis, improved systolic function, and mitigated subsequent death [19]. However, whether FGF-9 generates neovascularization in the post-MI infarcted diabetic heart remains elusive. Within the current study, we hypothesized that transplanted FGF-9 would inhibit vessel and capillary apoptosis and activate endogenous $\mathrm{c}-\mathrm{Kit}^{+\mathrm{ve}}$ cells for their differentiation into VSM and EC types, contributing to neovascularization in the post-MI nondiabetic and diabetic heart.

\section{Materials and Methods}

2.1. MI and FGF-9 Administration. MI was generated in diabetic $\mathrm{db} / \mathrm{db}$ and $\mathrm{C} 57 \mathrm{BL} / 6$ mice (8-12 weeks old) as approved by the University of Central Florida Institutional Animal Care and Use Committee (IACUC) and as previously detailed [20, 21]. $\mathrm{db} / \mathrm{db}$ and $\mathrm{C} 57 \mathrm{BL} / 6$ animals were divided independently into three groups ( $n=7-9$ animals/group): sham, MI, and MI + FGF-9 (1 ng/10 $\mu \mathrm{L} 0.1 \%$ BSA in $1 \mathrm{X}$ PBS). In brief, mice were anesthetized with isoflurane and a left thoracotomy was performed. The left descending coronary artery was visualized and permanently ligated. In the MI + FGF-9 group, FGF-9 was delivered via two injections (1 ng FGF-9/injection prepared as aforementioned for a total dose of 2 ng FGF-9) into the peri-infarct region using a 29-gauge floating needle. Sham operated animals underwent all surgical protocols with permanent artery ligation excluded. On day 14 postMI, animals were sacrificed using $4 \%$ inhalatory isoflurane for 10 mins followed by cervical dislocation. Hearts were subsequently removed and preserved for further analysis.

2.2. Apoptotic Vascular Cell Identification. All protocols for VSM and endothelial cell immunostaining were carried out as previously described [22]. In brief, $5 \mu \mathrm{m}$ serial sections were cut at the midpapillary level from paraffin embedded heart sections. Heart sections were deparaffinized in xylene, rehydrated by sequential incubation in $100 \%, 95 \%, 70 \%$, and $30 \%$ alcohol, and permeabilized with proteinase K (Sigma Aldrich cat \# P6556-10 mg, $25 \mu \mathrm{g} / \mathrm{mL}$ in $100 \mathrm{mM}$ Tris $\mathrm{HCl}$, cat \# BP152-5, Fisher Scientific). Apoptotic nuclei were visualized using a TUNEL assay (TMR red, cat \# 12156792910, Roche Applied Bio Sciences) as previously reported [22]. After TUNEL staining, heart sections were costained overnight with primary antibodies against anti-SM $\alpha$-actin (vascular smooth muscle cells, 1:100, cat \# A2172-0.2 mL, Sigma Aldrich) followed by an antimouse antibody (M.O.M. kit, cat \# FMK-2201, Vector laboratories). To detect endothelial cells post-TUNEL staining, heart sections were incubated with anti-CD31 (1:100, cat \# 250589, Abbiotec) followed by incubation with goat antirabbit IgG-FITC secondary antibodies (1:30, cat \# sc-2012, Santa Cruz). Sections were then mounted with Vectashield (Vector Laboratories, cat \# $\mathrm{H}-1200)$ containing DAPI and viewed under fluorescent and confocal microscopy. Left ventricular vessels within the periinfarcted area of the left ventricle, containing colocalized SM $\alpha$-actin and TUNEL or anti-CD31 and TUNEL, were considered apoptotic and quantified in 1-2 sections from $n=$ 5-6 animals/group.

2.3. c-Kit Activation and Differentiation. As previously reported, sections were deparaffinized in xylene, rehydrated in alcohol, washed with distilled water and PBS, and covered with $10 \%$ normal goat serum (NGS, cat \# s-1000, Vector Laboratories) for one hour to prevent nonspecific binding $[22,23]$. Heart sections were incubated for one hour with mouse monoclonal primary antibodies against $\mathrm{c}$-Kit (1:20, cat \# sc-365504, Santa Cruz) and costained with anti-SM $\alpha$-actin (1:15, cat \# A2172-0.2 mL, Sigma Aldrich) or anti-CD31 (1:20, cat \# sc-46694 Santa Cruz). Sections were then incubated with appropriate secondary antibodies (Alexa Fluor 568- or 488-conjugated goat antimouse IgG, cat \# A11019, Invitrogen) for one hour. Following secondary antibody incubation, stained heart sections were mounted with Vectashield antifade medium (cat \# H-1200, Vector Laboratories) containing DAPI and examined under confocal microscopy for photomicrographs and quantitative analysis. For quantitative analysis, four images per section (1-2 sections, $n=5$ animals/group) within the peri-infract region of the left ventricle were used to obtain the average number of c-kit cells positive for SM $\alpha$-actin or CD31.

2.4. Histological Quantification of Ventricular Vasculature. To visualize and quantify ventricular vasculature, prepared heart sections were stained with Masson's trichrome as previously described [6]. The number of vessels (in the peri-infarct region of the left ventricle) was quantified in 1-2 sections from $n=6-8$ animals per group using an Olympus microscope at 20x magnification.

2.5. Cardiac Function. Left ventricular function was determined using a Phillips Sonos 5500 ultrasound system. Animals were anesthetized with isoflurane and placed in the supine position on a controlled heating pad. Using a 15$6 \mathrm{~L}$ hockey-stick transducer, two-dimensional images were recorded and M-mode frames were used to measure left ventricular internal dimension-diastole (LVIDd), left ventricular internal dimension-systole (LVIDs), fractional shortening (FS, [(LVIDd - LVIDs)/LVIDd] × 100), left ventricular volume at end diastole (EDV), left ventricular volume at end systole (ESV), and ejection fraction (EF, [(EDV - ESV)/EDV] $\times 100)$ in the short-axis view at the midpapillary muscle level.

2.6. Data Analysis. All values are presented as a mean \pm SEM. Analysis of data was performed using one-way ANOVA followed by the Tukey post hoc test using SigmaStat. Statistical significance was assigned when $p<0.05$. 


\section{Results}

3.1. FGF-9 Prevents Vessel Apoptosis Post-MI. Representative photomicrographs illustrating vascular apoptosis are shown in Figure 1(a) for C57BL/6 (A-L) and $\mathrm{db} / \mathrm{db}(\mathrm{M}-\mathrm{X})$ mice with TUNEL positive nuclei in red (A, E, I, M, Q, and U), SM $\alpha$-actin in green (B, F, J, N, R, and V), total nuclei stained blue with DAPI (C, G, K, O, S, and W), and merged images (D, H, L, P, T, and X). The boxes on the top right panel of Figure 1(a) are enhanced images from each group to demonstrate the colocalization of TUNEL, SM $\alpha$-actin, and DAPI within a single vessel. Quantification of apoptotic vessels suggests vascular apoptosis is significantly enhanced in $\mathrm{C} 57 \mathrm{BL} / 6$ and $\mathrm{db} / \mathrm{db}$ mouse hearts post-MI relative to sham operated C57BL/6 and db/db mice $(p<0.05$, Figure $1(\mathrm{~b}))$. Importantly, FGF-9 treatment post-MI dramatically mitigated vessel apoptosis in both $\mathrm{C} 57 \mathrm{BL} / 6$ and $\mathrm{db} / \mathrm{db}$ mice compared to their MI operated counterparts $(p<0.05$, Figure 1(b)). However, no statistical significance was noted between $\mathrm{C} 57 \mathrm{BL} / 6$ and $\mathrm{db} / \mathrm{db}$ vasculature apoptosis outcomes in sham, MI, or MI + FGF-9 groups (Figure 1(b)).

3.2. FGF-9 Reduces Capillary Apoptosis following MI. Representative photomicrographs demonstrating capillary apoptosis are shown in Figure 2(a) for C57BL/6 (A-L) and db/db $(\mathrm{M}-\mathrm{X})$ mice with TUNEL positive nuclei in red (A, E, I, M, $\mathrm{Q}$, and $\mathrm{U}), \mathrm{CD} 31$ in green (B, F, J, N, R, and V), total nuclei stained blue with DAPI (C, G, K, O, S, and W), and merged images (D, H, L, P, T, and X). The smaller boxes (D, H, L, P, T, and $\mathrm{X}$ ) are enhanced images shown to illustrate colocalization of TUNEL, CD31, and DAPI within a single vessel. Endothelial cell death, as evidenced by TUNEL and CD31 positive cells, was significantly elevated in $\mathrm{C} 57 \mathrm{BL} / 6$ and $\mathrm{db} / \mathrm{db}$ mice post-MI $(p<0.05$, Figure $2(\mathrm{~b}))$. However, treatment with FGF-9 dramatically abrogated capillary apoptosis in postMI nondiabetic and diabetic mice $(p<0.05$, Figure $2(b))$. Moreover, endothelial cell death results were not significantly different between $\mathrm{C} 57 \mathrm{BL} / 6$ and $\mathrm{db} / \mathrm{db}$ mice within the same treatment group (Figure 2(b)).

3.3. FGF-9 Promotes c-Kit Activation and VSM Differentiation in Post-MI C57BL/6 and db/db Mice. The effect of FGF-9 on endogenous cardiac $\mathrm{c}-\mathrm{Kit}^{+\mathrm{ve}}$ progenitor cell activation and vascular smooth muscle cell differentiation was examined for all control and experimental animal groups. Representative images in Figure 3(a) demonstrate $\mathrm{c}-\mathrm{Kit}^{+\mathrm{ve}}$ progenitor cells in red (A, E, I, M, Q, and U), SM $\alpha$-actin VSM cells in green $(\mathrm{B}, \mathrm{F}, \mathrm{J}, \mathrm{N}, \mathrm{R}$, and $\mathrm{V})$, total nuclei in blue $(\mathrm{C}, \mathrm{G}, \mathrm{K}, \mathrm{O}, \mathrm{S}$, and $\mathrm{W}$ ), and merged images (D, H, L, P, T, and X) for C57BL/6 and $\mathrm{db} / \mathrm{db}$ groups. Enlarged images depicting colocalization of $\mathrm{c}-\mathrm{Kit}^{+\mathrm{ve}}$ and SM $\alpha$-actin ${ }^{+\mathrm{ve}}$ cells are presented in yellow boxes in Figure 3(a) (D, H, L, P, T, and X). Following MI, c-kit activation and VSM cell differentiation were not significantly different compared to the control group in both nondiabetic and diabetic mice (Figure 3(b)). However, when additionally treated with FGF-9 post-MI, the number of c-Kit ${ }^{+\mathrm{ve}} / \mathrm{SM} \alpha$ actin $^{\text {+ve }}$ cells was significantly enhanced in C57BL/6 and $\mathrm{db} / \mathrm{db}$ mice compared to their MI-alone counterparts $(p<$ 0.05 , Figure 3(b)).
3.4. FGF-9 Enhances c-Kit Activation and EC Differentiation in Infarcted Myocardium. To assess the effects of FGF-9 on ckit activation and endothelial cell differentiation in infarcted nondiabetic and diabetic myocardium, sections were double labeled with c-Kit and CD31. Representative images are shown in Figure 4(a) demonstrating $\mathrm{c}-\mathrm{Kit}^{+\mathrm{ve}}$ cells in red (A, $\mathrm{E}, \mathrm{I}, \mathrm{M}, \mathrm{Q}$, and $\mathrm{U}), \mathrm{CD} 31^{\text {+ve }}$ cells in green $(\mathrm{B}, \mathrm{F}, \mathrm{J}, \mathrm{N}, \mathrm{R}$, and $\mathrm{V}$ ), total nuclei in blue $(\mathrm{C}, \mathrm{G}, \mathrm{K}, \mathrm{O}, \mathrm{S}$, and $\mathrm{W})$, and merged images (D, H, L, P, T, and X). The number of cells positive for $\mathrm{c}$-Kit and CD31 in heart sections from each group was quantified and our data suggests that progenitor cell activation and endothelial cell differentiation were unchanged following $\mathrm{MI}$ in $\mathrm{C} 57 \mathrm{BL} / 6$ and $\mathrm{db} / \mathrm{db}$ myocardium compared to sham counterparts (Figure 4(b)). Moreover, FGF-9 treated post-MI hearts show an increase in number of $\mathrm{c}-\mathrm{Kit}^{+\mathrm{ve}} / \mathrm{CD} 31^{+\mathrm{ve}}$ cells was significantly enhanced in both nondiabetic and diabetic mice compared to sham operated mice $(p<0.05$, Figure $4(\mathrm{~b}))$. Notably, the outcome of FGF9 administration on $\mathrm{c}-\mathrm{Kit}$ activation and EC differentiation was similar between C57BL/6 and db/db mice (Figure 4(b)). Collectively, our data implies FGF-9 treatment promotes endogenous c-Kit progenitor cell activation and differentiation into VSM cells and ECs in nondiabetic and diabetic infarcted myocardium.

3.5. Transplanted FGF-9 Promotes Vessel Formation in $C 57 B L / 6$ and $d b / d b$ Mice Post-MI. To determine the effects of FGF-9 administration on coronary artery formation, the total number of small, medium, and large vessels was counted from Masson's trichrome stained heart sections as depicted in Figure 5(a). In both nondiabetic and diabetic myocardium, the number of total vessels was significantly decreased following MI compared to the sham groups $(p<0.05$, Figure 5(b)). However, following FGF-9 treatment, a significant increase in the total number of myocardial vessels was significantly enhanced in the MI + FGF-9 group compared with the MI group in $\mathrm{C} 57 \mathrm{BL} / 6$ and $\mathrm{db} / \mathrm{db}$ mice $(p<0.05$, Figure $5(\mathrm{~b}))$.

3.6. FGF-9 Improved Left Ventricular Cardiac Function Post$M I$ in $C 57 B L / 6$ and $d b / d b$ Mice. Cardiac function was assessed two weeks after coronary artery ligation or sham operations via echocardiography for all control and experimental animals. All raw and calculated cardiac functional data are presented in Figures 6(a)-6(g). Following MI, left ventricular output, as indicated by fractional shortening and ejection fraction, was significantly hindered following MI surgery relative to sham controls in both $\mathrm{C} 57 \mathrm{BL} / 6$ and $\mathrm{db} / \mathrm{db}$ mice $(p<0.05$, Figures $6(\mathrm{~d})$ and $6(\mathrm{~g})$, resp.). Importantly, a significant increase in left ventricular function was observed following FGF-9 administration in nondiabetic and diabetic mice relative to their MI group counterparts $(p<0.05$, Figures 6(d) and 6(g)).

\section{Discussion}

After MI, myocardial angiogenesis is a reparative process responsible for resupplying ischemic tissue with oxygen and nutrients and is ultimately a key determinant of infarction and pathophysiological disease progression $[6,24]$. 


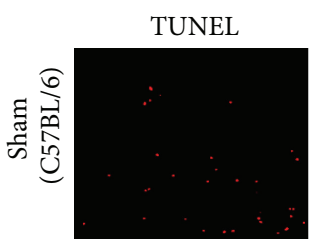

(A)

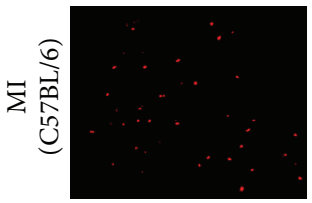

(E)

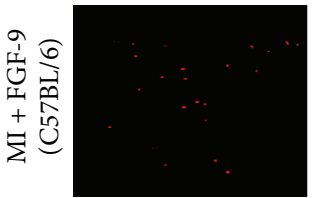

(I)

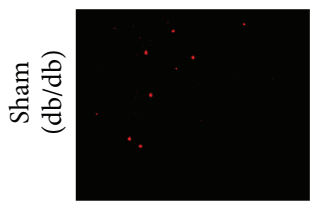

(M)

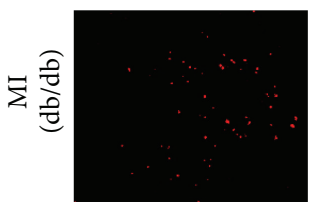

(Q)

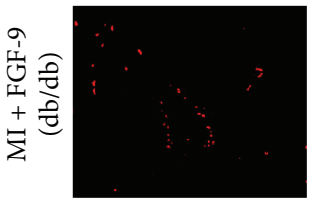

(U)
SM $\alpha$-actin

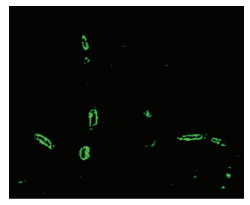

(B)

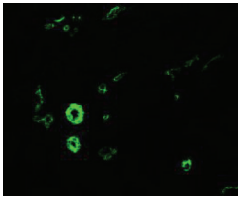

(F)

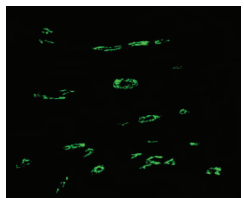

(J)

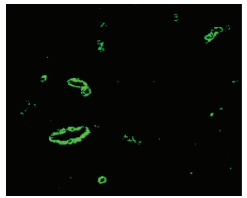

(N)

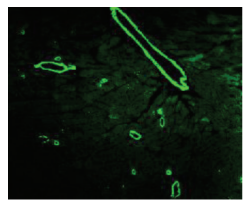

(R)

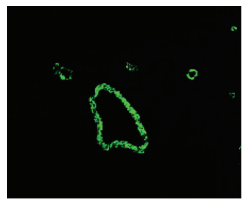

(V)

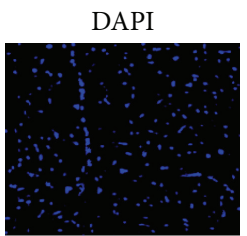

(C)

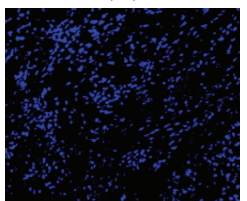

(G)

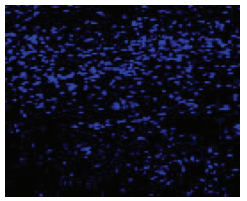

(K)

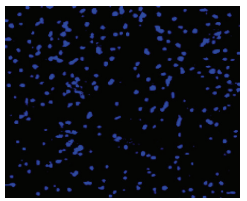

(O)

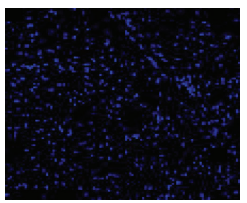

(S)

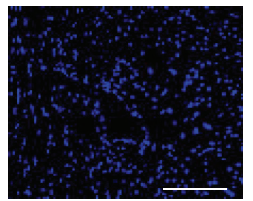

(W)

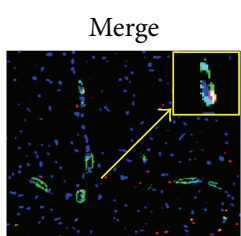

(D)

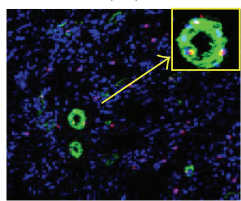

(H)

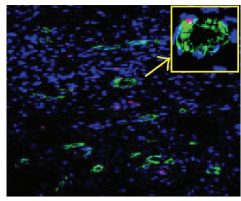

(L)

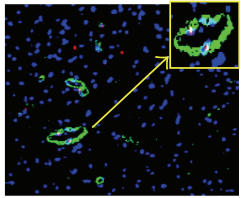

(P)

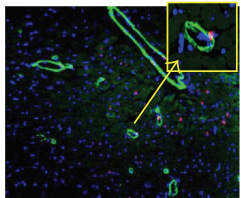

(T)

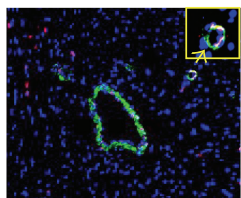

(X)

(a)

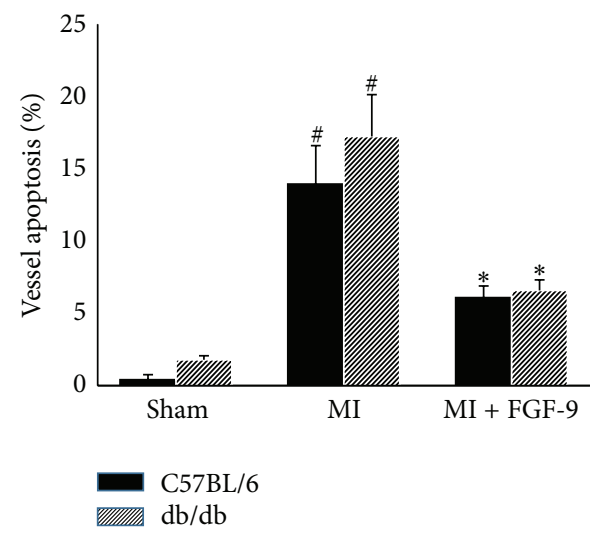

(b)

FIGURE 1: FGF-9 blunts vessel apoptosis in the diabetic infarcted heart. Representative photomicrographs illustrating vascular apoptosis are depicted in (a) for C57BL/6 (A-L) and db/db (M-X) mice with TUNEL positive nuclei in red (A, E, I, M, Q, and U), SM $\alpha$-actin ${ }^{+v e}$ cells in green (B, F, J, N, R, and V), total nuclei stained blue with DAPI (C, G, K, O, S, and W), and merged images (D, H, L, P, T, and X). The boxes on the top right panel of (a) are enhanced images from each group to demonstrate the colocalization of TUNEL, SM $\alpha$-actin, and DAPI within a single vessel. Scale bar $=100 \mu \mathrm{m}$. (b) Histogram of quantitative vessels apoptosis in infarcted C57BL/6 and db/db mice. ${ }^{\#} p<0.05 \mathrm{versus}$ sham and ${ }^{*} p<0.05$ versus MI. 


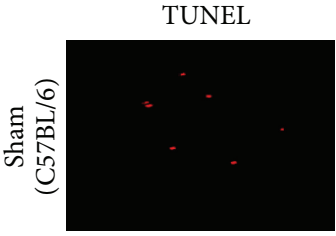

(A)

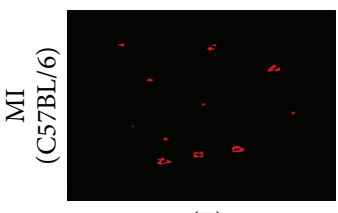

(E)

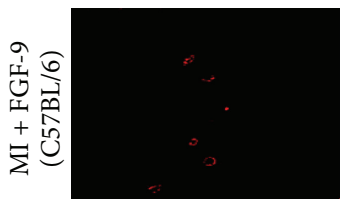

(I)

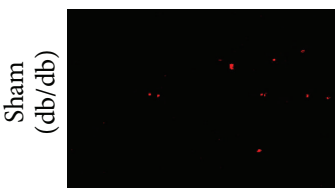

(M)

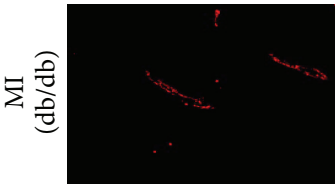

(Q)

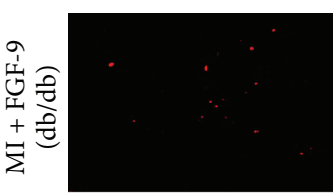

(U)
CD31

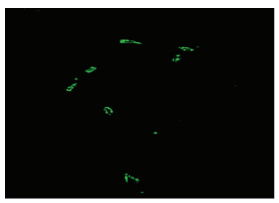

(B)

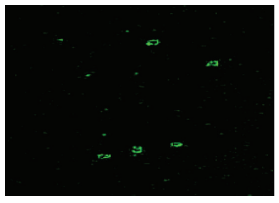

(F)

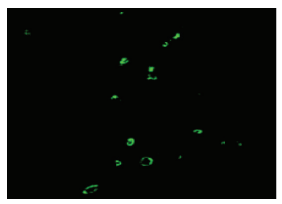

(J)

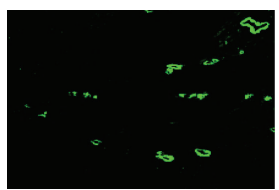

$(\mathrm{N})$

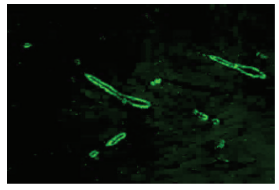

(R)

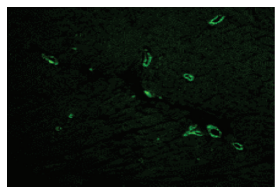

(V)

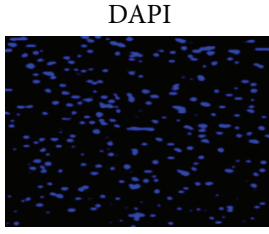

(C)

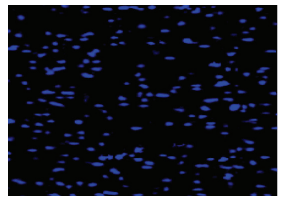

(G)

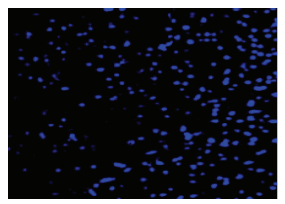

(K)

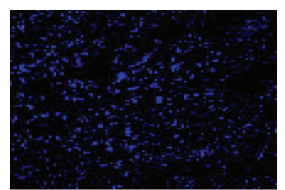

(O)

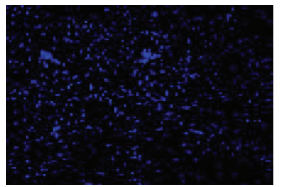

(S)

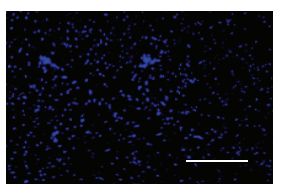

(W)

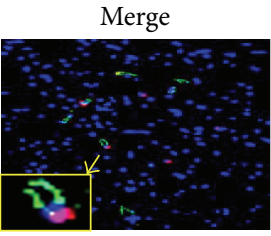

(D)

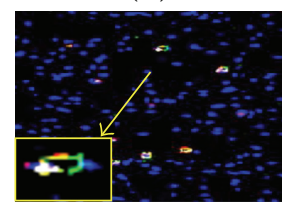

(H)

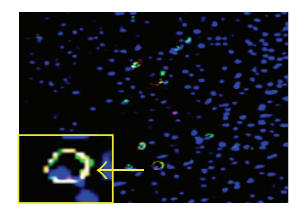

(L)

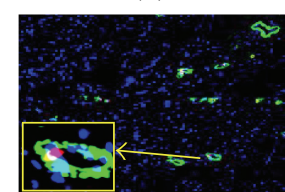

(P)

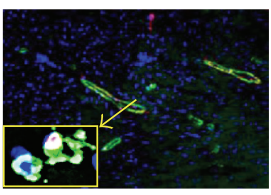

(T)

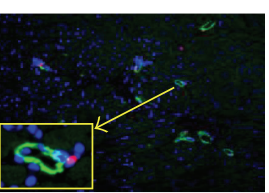

(X)

(a)

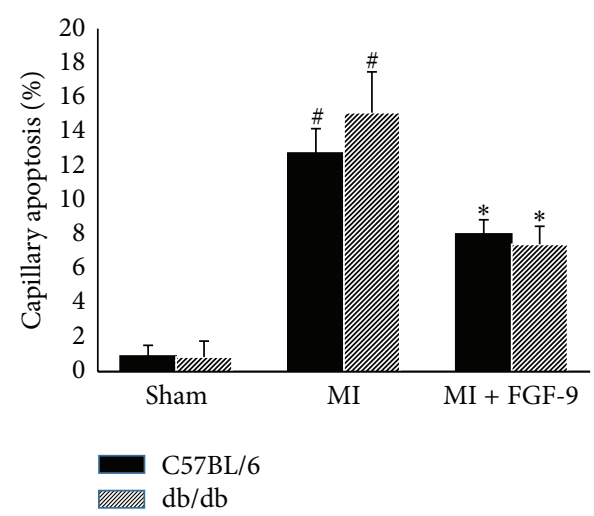

(b)

FIGURE 2: Effects of FGF-9 on capillary apoptosis following MI in C57BL/6 and db/db mice. Representative images demonstrating capillary apoptosis are illustrated in (a) for C57BL/6 (A-L) and db/db (M-X) mice with TUNEL positive nuclei in red (A, E, I, M, Q, and U), CD31 $1^{+v e}$ cells in green (B, F, J, N, R, and V), total nuclei stained blue with DAPI (C, G, K, O, S, and W), and merged images (D, H, L, P, T, and X). The smaller boxes (D, H, L, P, T, and X) are enhanced images shown to illustrate colocalization of TUNEL, CD31, and DAPI within a single vessel. Scale bar $=100 \mu \mathrm{m}$. (b) Quantitative data suggest FGF-9 inhibits capillary apoptosis following MI in C57BL/6 and db/db mice. ${ }^{\#} p<0.05$ versus sham and ${ }^{*} p<0.05$ versus MI. 


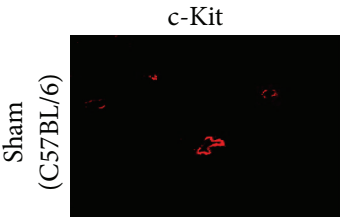

(A)

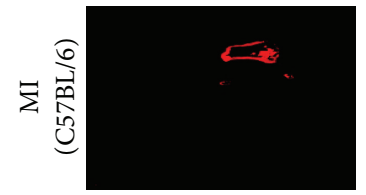

(E)

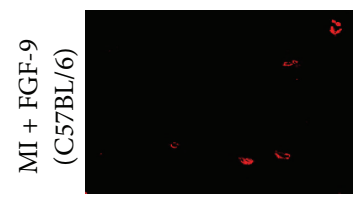

(I)

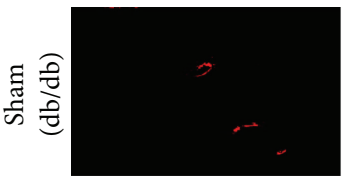

(M)

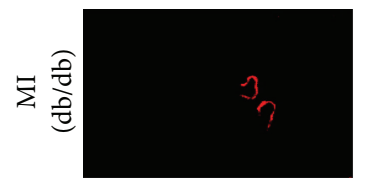

(Q)

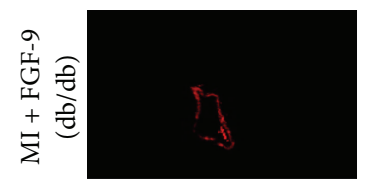

(U)
$\operatorname{SM} \alpha$-actin

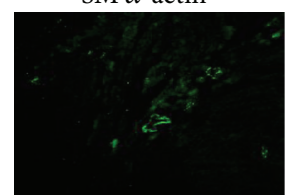

(B)

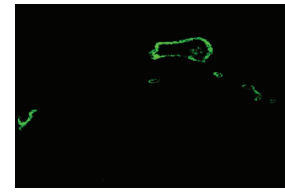

(F)

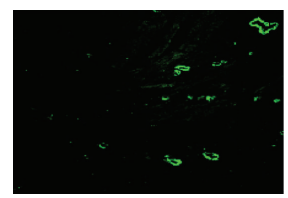

(J)

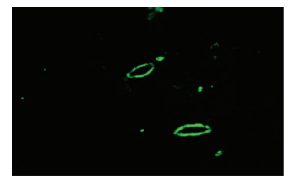

(N)

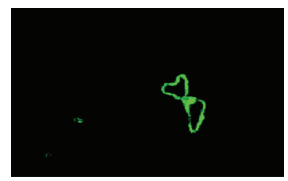

(R)

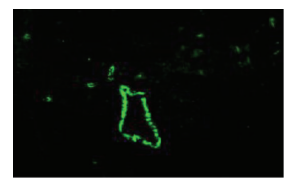

(V)

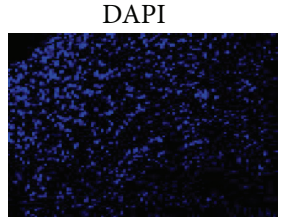

(C)

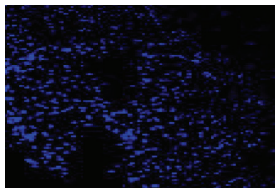

(G)

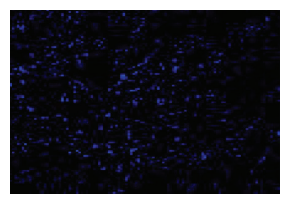

(K)

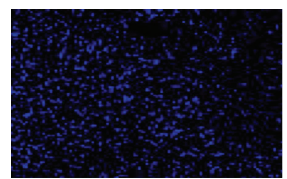

(O)

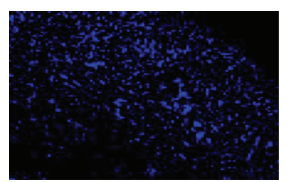

(S)

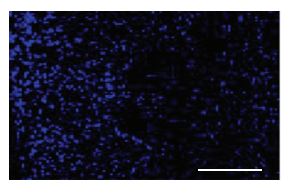

(W)

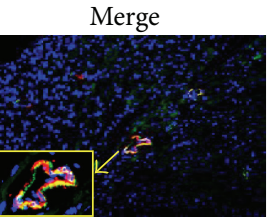

(D)

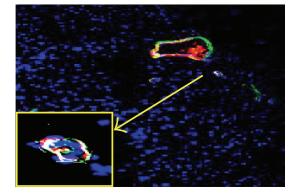

(H)

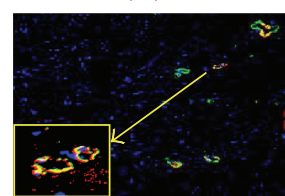

(L)

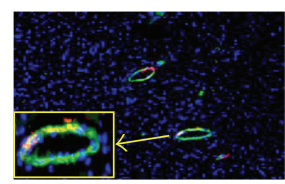

(P)

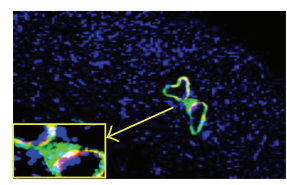

(T)

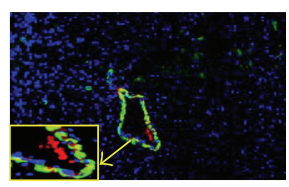

(X)

(a)

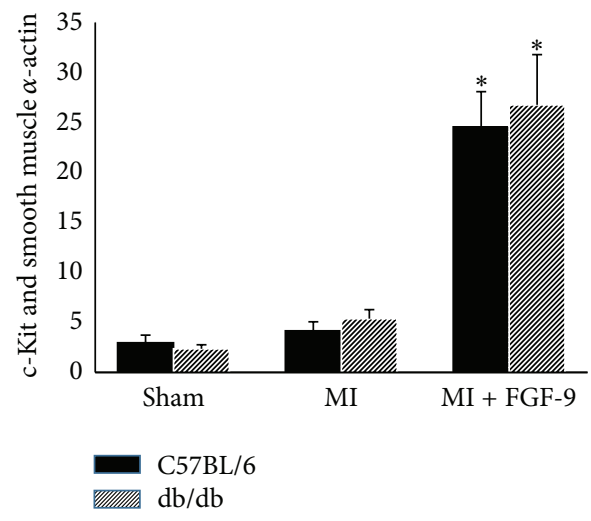

(b)

FIGURE 3: FGF-9 enhances c-Kit activation and vascular smooth muscle cell differentiation in post-MI C57BL/6 and db/db mice. Representative photomicrographs in (a) depict c-Kit ${ }^{\text {tve }}$ progenitor cells in red (A, E, I, M, Q, and U), SM $\alpha$-actin VSM cells in green (B, $\mathrm{F}, \mathrm{J}, \mathrm{N}, \mathrm{R}$, and V), total nuclei in blue (C, G, K, O, S, and W), and merged images $(\mathrm{D}, \mathrm{H}, \mathrm{L}, \mathrm{P}, \mathrm{T}$, and $\mathrm{X})$ for C57BL/6 and db/db groups. Enlarged images depicting colocalization of c-Kit ${ }^{+v e}$ and SM $\alpha$-actin ${ }^{+v e}$ cells are presented in yellow boxes in (a) (D, H, L, P, T, and X). Scale bar $=100 \mu \mathrm{m}$. (b) The number of c-Kit ${ }^{+v e} / \mathrm{SM} \alpha$-actin ${ }^{+v e}$ cells is significantly enhanced in the MI + FGF-9 group compared to the MI group in both C57BL/ 6 and db/db infarcted hearts. ${ }^{*} p<0.05$ versus MI. VSM = vascular smooth muscle. 


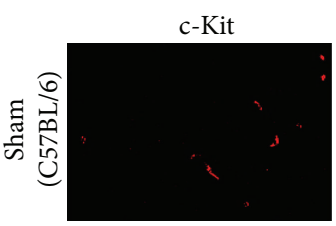

(A)

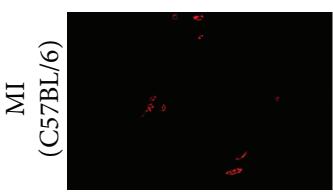

(E)

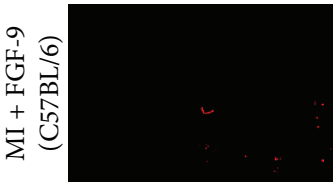

(I)

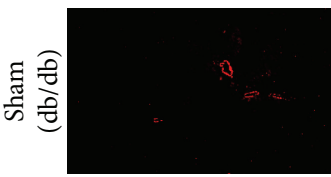

(M)

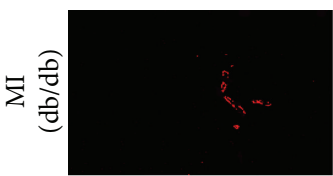

(Q)

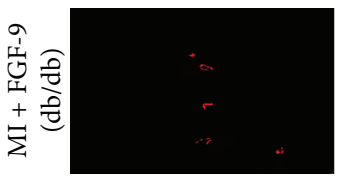

(U)

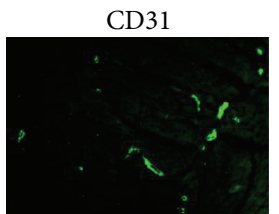

(B)

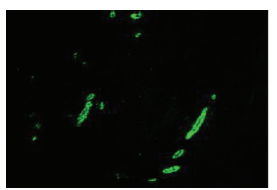

(F)

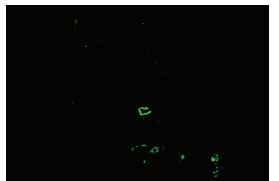

(J)

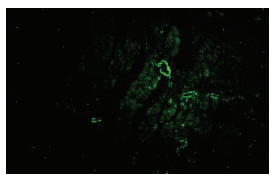

(N)

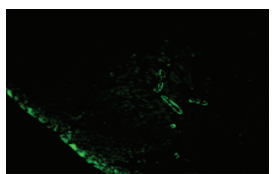

(R)

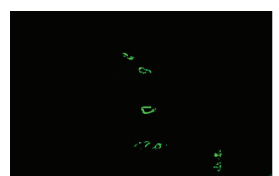

(V)

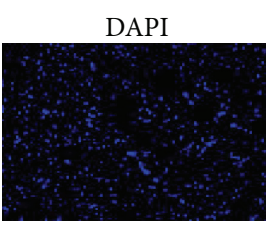

(C)

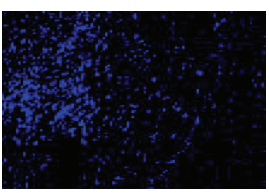

(G)

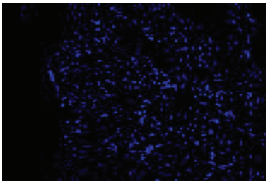

(K)

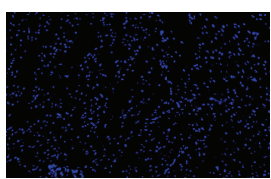

(O)

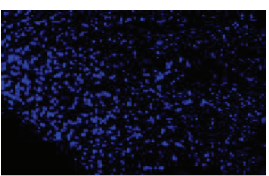

(S)

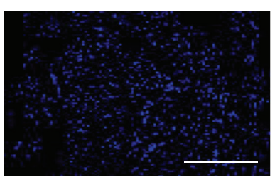

(W)

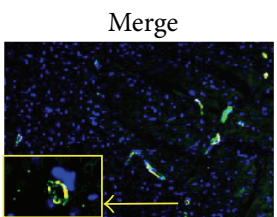

(D)

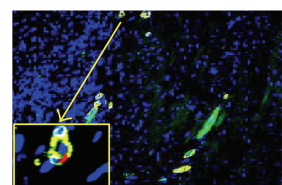

(H)

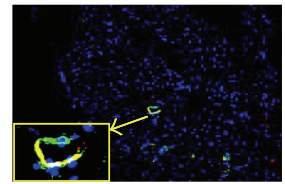

(L)

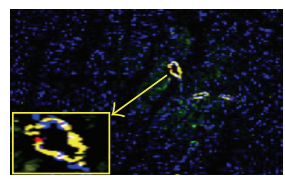

(P)

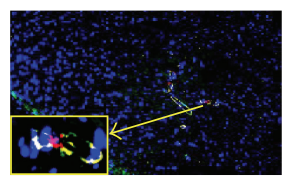

(T)

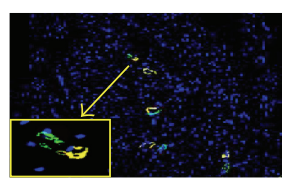

(X)

(a)

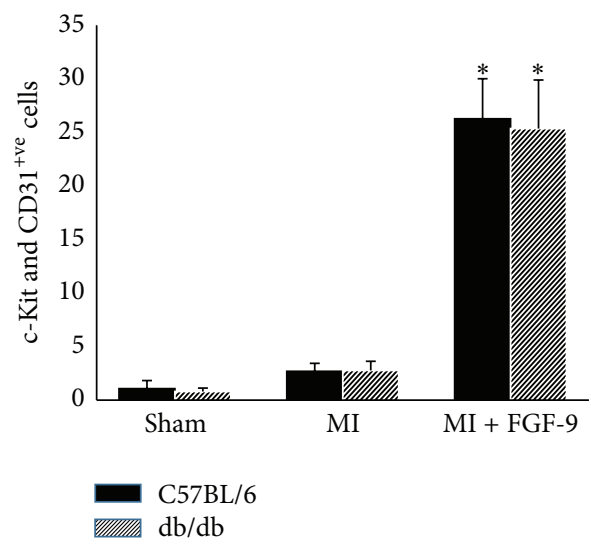

(b)

FIGURE 4: FGF-9 promotes c-Kit activation and endothelial cell differentiation in post-MI C57BL/6 and db/db mice. Representative images are shown in (a) demonstrating $\mathrm{c}-\mathrm{Kit}^{+\mathrm{ve}}$ cells in red (A, E, I, M, Q, and U), CD31 ${ }^{\text {+ve }}$ cells in green (B, F, J, N, R, and V), total nuclei in blue $\left(\mathrm{C}, \mathrm{G}, \mathrm{K}, \mathrm{O}, \mathrm{S}\right.$, and W), and merged images $\left(\mathrm{D}, \mathrm{H}, \mathrm{L}, \mathrm{P}, \mathrm{T}\right.$, and X). Enlarged images depicting colocalization of c-Kit ${ }^{+\mathrm{ve}}$ and CD31 ${ }^{+\mathrm{ve}}$ cells are presented in yellow boxes in (a) (D, H, L, P, T, and X). Scale bar $=100 \mu \mathrm{m}$ (b) Histogram of quantified c-Kit ${ }^{+\mathrm{ve}} / \mathrm{CD} 31^{+\mathrm{ve}}$ cells in C57BL/6 and $\mathrm{db} / \mathrm{db}$ infarcted hearts suggest FGF-9 enhances c-Kit activation and EC differentiation. ${ }^{*} p<0.05$ versus MI. EC = endothelial cell. 


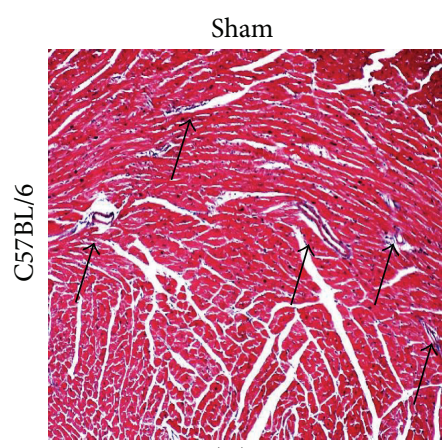

(A)

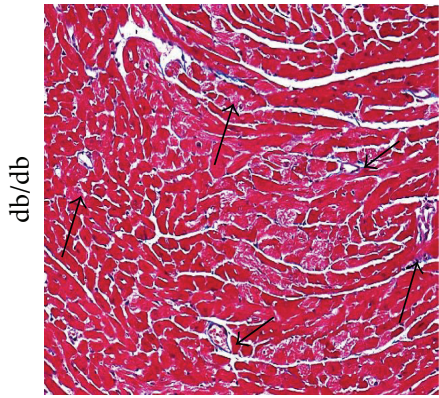

(D)
MI

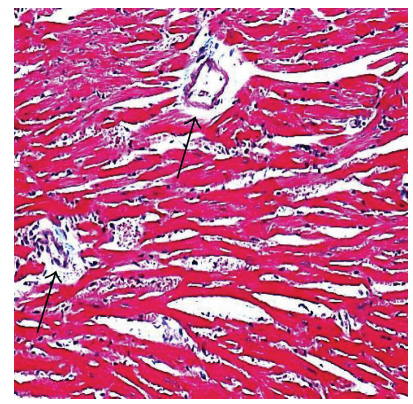

(B)

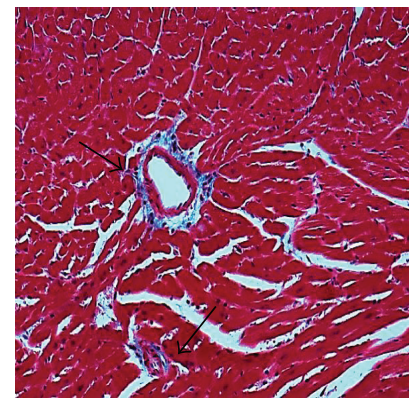

(E)

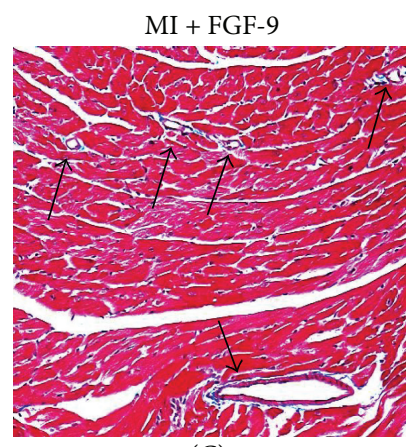

(C)

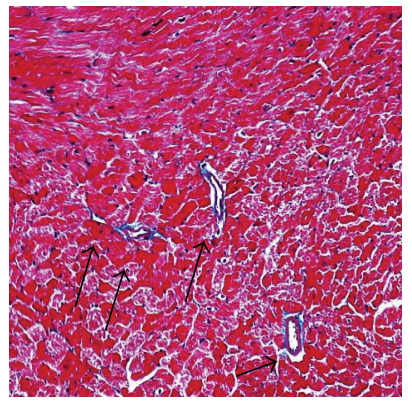

(F)

(a)

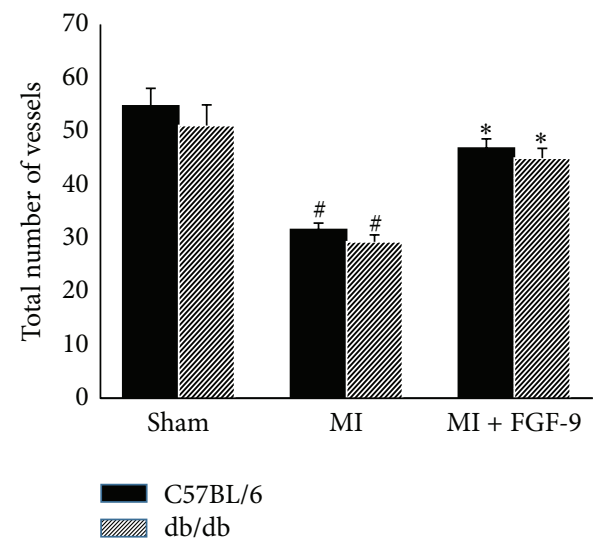

(b)

FIGURE 5: FGF-9 enhances vessel formation in postinfarcted nondiabetic and diabetic hearts. (a) Representative Masson's trichrome stained myocardial images for all control and experimental groups depicting blood vessels as indicated by the black arrows. (b) Data suggest that, following MI, the total number of vessels in the infarcted heart is significantly decreased whereas, following FGF- 9 treatment, the total number of vessels is significantly increased relative to the MI group for both C57BL/6 and db/db mice. ${ }^{*} p<0.05$ versus sham and ${ }^{*} p<0.05 \mathrm{versus}$ MI.

Well documented, NIDDM is associated with an increased risk for developing secondary cardiovascular complications including coronary arterial disease (CAD), stroke, and peripheral arterial disease (PAD), all of which involve impaired angiogenesis [25-28]. Previous data have suggested that maladaptive myocardial angiogenesis in the context of diabetic patients is consequent, in part, to disrupted angiopoietins/Tie-2 signaling as well as diminished vascular endothelial growth factor (VEGF) expression [8-10]. Attempts to improve neoangiogenic outcomes post-MI have become an innovative target for many investigators using various proteins/small molecules including Apelin (APLN), thioredoxin-interacting protein (TXNIP) inhibitors, and peroxisome proliferator-activated receptors (PPARs) agonists, as well as various stem cell types [8,29-34]. Notably, previously reported data have suggested that FGF-9 plays a role in coronary neovasculogenesis as well as enhancing microvessel density in the post-MI heart $[13,19]$. However, there is no available data with regards to FGF-9 and angiogenesis in the postinfarcted diabetic heart nor has any attempt been made to elucidate mechanisms by which FGF-9 enhances post-MI angiogenesis, including the involvement of c-Kit stem cell activation. In the present study, we have evaluated the effects of FGF-9 treatment on vessel apoptosis, $\mathrm{c}-\mathrm{Kit}^{+\mathrm{ve}}$ stem cell 


\begin{tabular}{lcccccc}
\hline Group & LVIDd & LVIDs & EDV & ESV & FS\% & EF\% \\
\hline Sham (C57BL/6) & $2.68 \pm 0.11$ & $1.33 \pm 0.07$ & $29.37 \pm 3.21$ & $4.6 \pm 0.62$ & $53.67 \pm 1.26$ & $85.39 \pm 1.08$ \\
Sham (db/db) & $2.86 \pm 0.20$ & $2.86 \pm 0.2$ & $31.65 \pm 5.51$ & $7.5 \pm 1.48$ & $48.93 \pm 1.41$ & $78.80 \pm 1.22$ \\
MI (C57BL/6) & $3.29 \pm 0.19$ & $2.05 \pm 0.08$ & $46.18 \pm 3.15$ & $13.00 \pm 1.47$ & $33.89 \pm 0.9$ & $65.33 \pm 2.4$ \\
MI (db/db) & $3.22 \pm 0.13$ & $3.22 \pm 0.13$ & $44.97 \pm 3.96$ & $19.16 \pm 1.89$ & $31.31 \pm 1.55$ & $60.54 \pm 2.8$ \\
MI + FGF9 (C57BL/6) & $2.55 \pm 0.14$ & $1.52 \pm 0.07$ & $24.12 \pm 2.89$ & $6.42 \pm 0.75$ & $41.57 \pm 1.64$ & $74.45 \pm 1.88$ \\
MI + FGF9 (db/db) & $3.21 \pm 0.23$ & $3.21 \pm 0.23$ & $33.16 \pm 1.47$ & $9.4 \pm 0.16$ & $40.62 \pm 0.92$ & $73.15 \pm 1.09$ \\
\hline
\end{tabular}

(a)
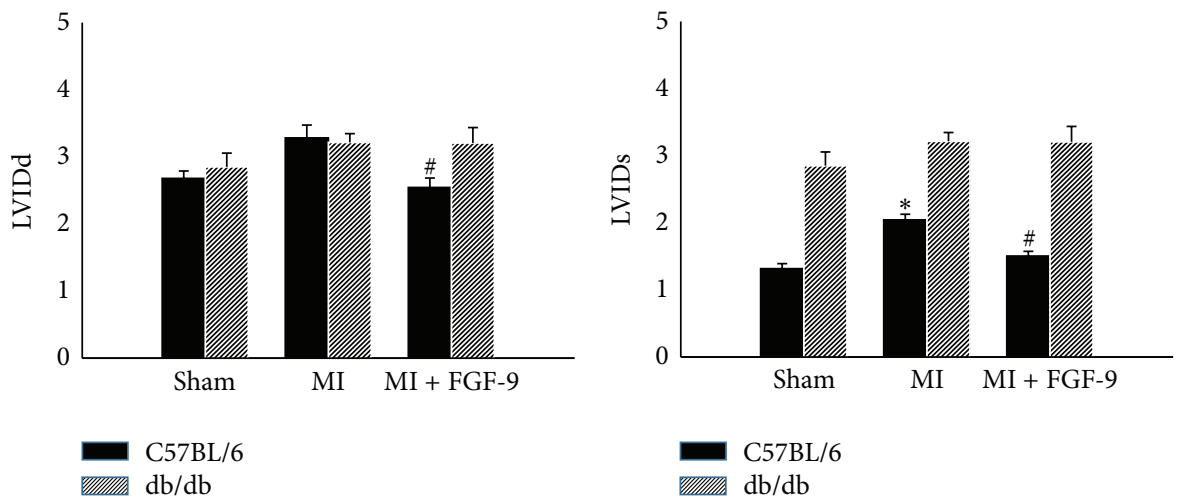

(b)

(c)
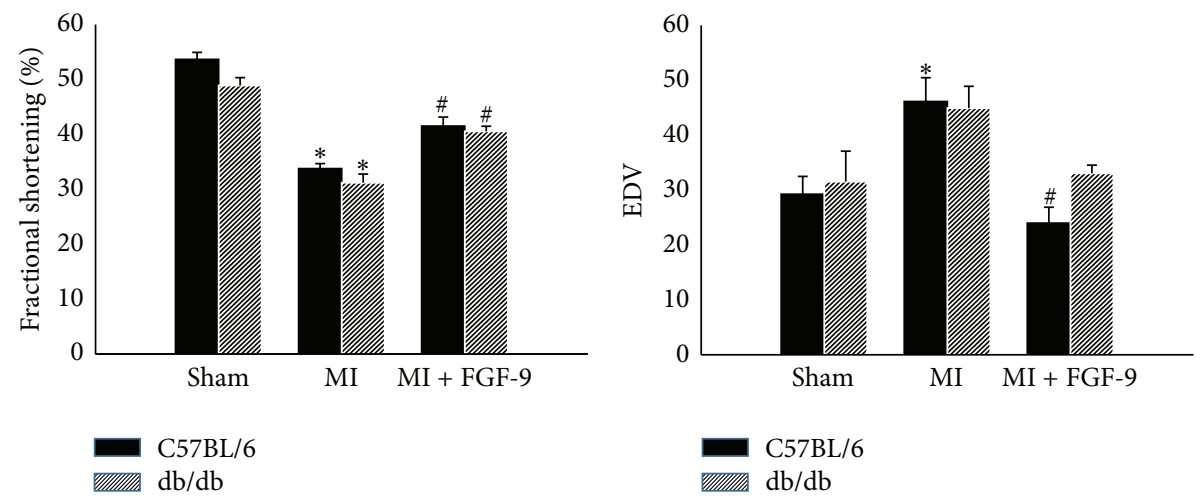

(d)

(e)
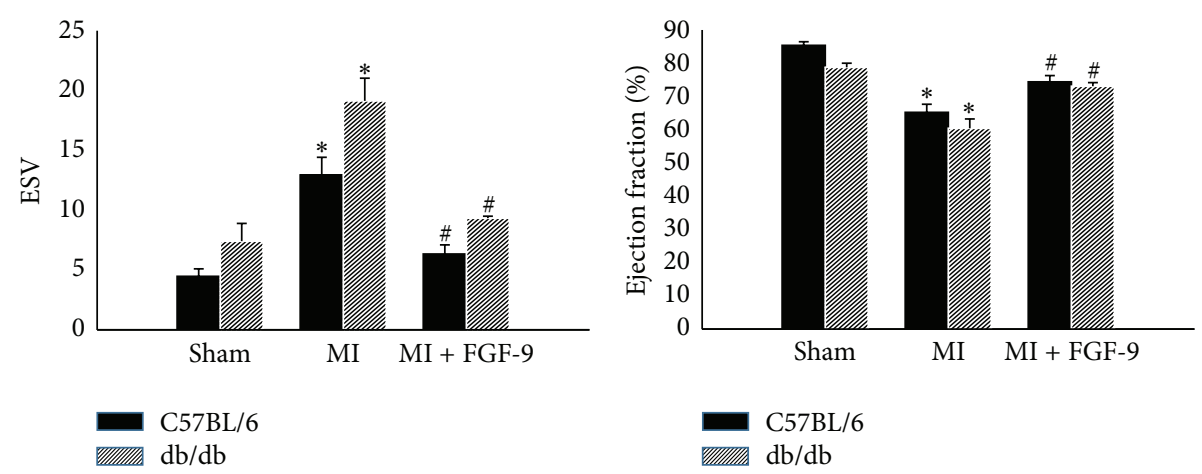

(f)

(g)

Figure 6: FGF-9 enhances left ventricular function in post-MI C57BL/6 and db/db mice. Two weeks following MI or sham surgery, heart function was assessed using transthoracic 2D echocardiography. (a) Table includes calculated cardiac functional data for all control and experimental groups. Histograms demonstrate quantitative analysis of (b) LVIDd, (c) LVIDs, (d) \% fractional shortening, (e) EDV, (f) ESV, and $(\mathrm{g}) \%$ ejection fraction. LVIDd = left ventricular internal dimension-diastole, LVIDs = left ventricular internal dimension-systole, FS = fractional shortening, EDV = left ventricular volume at end diastole, ESV = left ventricular volume at end systole, and $\mathrm{EF}=$ ejection fraction. ${ }^{\#} p<0.05$ versus sham and ${ }^{*} p<0.05$ versus MI. 
stimulation, new vessel formation, and cardiac function in post-MI nondiabetic and diabetic mice. To the best of our knowledge, this is the first investigation into the vascular impact propagated by FGF-9 in the post-MI NIDDM injured myocardium.

To elucidate the impact of FGF-9 on the postischemic revascularization process, we first wanted to assess the ability of FGF-9 to inhibit post-MI vascular apoptosis. Widely accepted, vascular apoptosis plays a pivotal role in the manifestation and progression of a myriad of post-MI cardiac adverse outcomes but has yet to be investigated with regards to FGF-9. As supported by many other investigations, vascular apoptosis was significantly enhanced in both nondiabetic and diabetic mice post-MI $[1,6,35]$. Importantly, treatment with FGF-9 post-MI significantly abrogated vasculature apoptosis. Although this is the first report indicating inhibited vasculature apoptosis in the post-MI heart via FGF-9, supporting evidence of cellular protection has been identified in other cell types including neurons and fetal gonocytes $[36,37]$. However, future studies are warranted to identify mechanisms by which FGF-9 provides vascular protection in the ischemic myocardium.

Evidence provided has suggested that neovascularization is a complex, multifactorial process involving proangiogenic growth factors including VEGF, granulocyte colony stimulating factor (G-CSF), insulin growth factor-1 (IGF-1), and stem cell factor (SCF) which in turn activate c-Kit stem cells and promote their differentiation into VSM cells and ECs [6]. With this aforementioned data in mind, we examined whether FGF-9 had any impact on c-Kit positive stem cell activation in the nondiabetic and diabetic infarcted heart. Importantly, a significant increase in c-Kit ${ }^{+v e}$ progenitor cells costained with SM $\alpha$-actin, identifying VSM cells, and c$\mathrm{Kit}^{+\mathrm{ve}}$ progenitor cells costained with CD31, identifying ECs, were identified following post-MI FGF-9 treatment. Of note, there was a slight increase in the amount of $\mathrm{c}-\mathrm{Kit}^{+\mathrm{ve}} / \mathrm{SM} \alpha$ actin $^{+v e}$ cells and $\mathrm{c}-\mathrm{Kit}^{+\mathrm{ve}} / \mathrm{CD} 31^{+\mathrm{ve}}$ cells in the MI group compared with sham, but this trend was not statistically significant. Although the exact reasons for this increase are unknown, we postulate that it may be consequent to post-MI microenvironmental alterations in cytokine/growth factor expression. Additionally, nondiabetic and diabetic mice had similar results regarding concentrations of $\mathrm{c}-\mathrm{Kit}^{+\mathrm{ve}} / \mathrm{SM} \alpha$ actin $^{+\mathrm{ve}}$ cells and $\mathrm{c}-\mathrm{Kit}^{+\mathrm{ve}} / \mathrm{CD} 31^{+\mathrm{ve}}$ cells in the infarcted heart. We suggest FGF-9 may promote neovascularization by inhibiting apoptosis and by acting directly on c-kit cells or enhancing proangiogenic factors that are disrupted in both nondiabetic and diabetic post-MI hearts. Altogether, our quantitative data suggest that endogenous c-Kit progenitor cells were activated and differentiated into neovascular cell types in the presence of FGF-9 in the infarcted heart. In this paper, we report a correlation between FGF-9 and c-Kit progenitor cell activation; however, future studies are necessitated to identify exact signaling pathways and cytokine/chemokine mediators, which drive FGF-9-induced neovascularization.

To verify enhanced neovascularization in the C57BL/6 and $\mathrm{db} / \mathrm{db}$ infarcted hearts, sections were stained with Masson's trichrome and the total numbers of vessels were counted for all control and experimental groups. Quantitative data suggest that there was significant increase in the total number of myocardial vessels following post-MI FGF-9 treatment compared to the MI group counterparts. Our data further corroborate our earlier findings of enhanced c-Kit progenitor cell activation and VSM cell and EC differentiation in the MI + FGF-9 group. Supportive data previously published demonstrate the ability of multiple stem cells (embryonic stem (ES) cells, mesenchymal stem cells (MSCs), and induced pluripotent stem (iPS) cells), to promote c-Kit progenitor cell activation and ultimately enhance angiogenesis and neovascularization in post-MI animal models [6, 22, 38-41].

Finally, we assessed the impact of neovascularization on the overall left ventricular output post-MI for all C57BL/6 and $\mathrm{db} / \mathrm{db}$ groups. Two weeks after MI, cardiac function was obtained by transthoracic 2D echocardiography. Functional data, including fractional shortening and ejection fraction, were significantly improved following FGF-9 treatment in infarcted nondiabetic and diabetic mice relative to MI counterparts. Collectively, our data suggests that FGF-9 inhibits vascular apoptosis and enhances angiogenesis, which contributes to an improvement in cardiac function following MI.

In conclusion, we report for the first time that FGF-9 prevents post-MI vascular apoptosis, enhances c-Kit activation and their differentiation into VSM cells and ECs, and improves overall cardiac function in C57BL/6 and $\mathrm{db} / \mathrm{db}$ mice. Our study provides evidence that FGF-9 enhances angiogenesis in infarcted myocardium and may have therapeutic potential for post-MI nondiabetic and diabetic patients. Moreover, we suggest that mechanisms of enhanced angiogenesis may deviate between diabetic and nondiabetic subjects and future studies are warranted to evaluate these differences including infarct size and augmented molecular signaling pathways as a result of FGF9 treatment.

\section{Conflict of Interests}

The authors declare that there is no conflict of interests regarding the publication of this paper.

\section{Funding}

This work was supported, in part, by grants from the National Institutes of Health [1R01HL090646-01 and 5R01HL09446702 to Dinender Singla].

\section{References}

[1] K. Y. Howangyin and J.-S. Silvestre, "Diabetes mellitus and ischemic diseases: molecular mechanisms of vascular repair dysfunction," Arteriosclerosis, Thrombosis, and Vascular Biology, vol. 34, no. 6, pp. 1126-1135, 2014.

[2] H. M. Smith, M. Hamblin, and M. F. Hill, "Greater propensity of diabetic myocardium for oxidative stress after myocardial infarction is associated with the development of heart failure," Journal of Molecular and Cellular Cardiology, vol. 39, no. 4, pp. 657-665, 2005. 
[3] G. Casella, S. Savonitto, F. Chiarella et al., "Clinical characteristics and outcome of diabetic patients with acute myocardial infarction. Data from the BLITZ-1 study," Italian Heart Journal, vol. 6 , no. 5, pp. 374-383, 2005.

[4] C. E. Glass, P. K. Singal, and D. K. Singla, "Stem cells in the diabetic infarcted heart," Heart Failure Reviews, vol. 15, no. 6, pp. 581-588, 2010.

[5] A. Araszkiewicz, M. Janus, M. Prech et al., "Relations of diabetes mellitus, microvascular reperfusion and left ventricular remodelling in patients with acute myocardial infarction treated with primary coronary intervention," Kardiologia Polska, vol. 72, no. 1, pp. 20-26, 2014.

[6] B. Yan, L. S. Abdelli, and D. K. Singla, "Transplanted induced pluripotent stem cells improve cardiac function and induce neovascularization in the infarcted hearts of $\mathrm{db} / \mathrm{db}$ mice," Molecular Pharmaceutics, vol. 8, no. 5, pp. 1602-1610, 2011.

[7] B. Yan and D. K. Singla, "Transplanted induced pluripotent stem cells mitigate oxidative stress and improve cardiac function through the akt cell survival pathway in diabetic cardiomyopathy," Molecular Pharmaceutics, vol. 10, no. 9, pp. 3425-3432, 2013.

[8] X. Hou, H. Zeng, X. He, and J.-X. Chen, "Sirt3 is essential for apelin-induced angiogenesis in post-myocardial infarction of diabetes," Journal of Cellular and Molecular Medicine, vol. 19, no. 1, pp. 53-61, 2015.

[9] J.-X. Chen and A. Stinnett, "Disruption of Ang-1/Tie-2 signaling contributes to the impaired myocardial vascular maturation and angiogenesis in type II diabetic mice," Arteriosclerosis, Thrombosis, and Vascular Biology, vol. 28, no. 9, pp. 1606-1613, 2008.

[10] Q.-H. Tuo, H. Zeng, A. Stinnett et al., "Critical role of angiopoietins/Tie-2 in hyperglycemic exacerbation of myocardial infarction and impaired angiogenesis," The American Journal of Physiology-Heart and Circulatory Physiology, vol. 294, no. 6, pp. H2547-H2557, 2008.

[11] A. Beenken and M. Mohammadi, "The FGF family: biology, pathophysiology and therapy," Nature Reviews Drug Discovery, vol. 8, no. 3, pp. 235-253, 2009.

[12] N. Itoh and D. M. Ornitz, "Fibroblast growth factors: from molecular evolution to roles in development, metabolism and disease," Journal of Biochemistry, vol. 149, no. 2, pp. 121-130, 2011.

[13] M. J. Frontini, Z. Nong, R. Gros et al., "Fibroblast growth factor 9 delivery during angiogenesis produces durable, vasoresponsive microvessels wrapped by smooth muscle cells," Nature Biotechnology, vol. 29, no. 5, pp. 421-427, 2011.

[14] D. Gay, O. Kwon, Z. Zhang et al., "Fgf9 from dermal $\gamma \delta \mathrm{T}$ cells induces hair follicle neogenesis after wounding," Nature Medicine, vol. 19, no. 7, pp. 916-923, 2013.

[15] U. Pirvola, X. Zhang, J. Mantela, D. M. Ornitz, and J. Ylikoski, "Fgf9 signaling regulates inner ear morphogenesis through epithelial-mesenchymal interactions," Developmental Biology, vol. 273, no. 2, pp. 350-360, 2004.

[16] Y. Lin, L. Chen, C. Lin, Y. Luo, R. Y. L. Tsai, and F. Wang, "Neuron-derived FGF9 is essential for scaffold formation of Bergmann radial fibers and migration of granule neurons in the cerebellum," Developmental Biology, vol. 329, no. 1, pp. 44-54, 2009.

[17] M. Harada, H. Murakami, A. Okawa et al., "FGF9 monomerdimer equilibrium regulates extracellular matrix affinity and tissue diffusion," Nature Genetics, vol. 41, no. 3, pp. 289-298, 2009.
[18] J. S. Colvin, R. P. Green, J. Schmahl, B. Capel, and D. M. Ornitz, "Male-to-female sex reversal in mice lacking fibroblast growth factor 9," Cell, vol. 104, no. 6, pp. 875-889, 2001.

[19] M. Korf-Klingebiel, T. Kempf, K.-D. Schlüter et al., "Conditional transgenic expression of fibroblast growth factor 9 in the adult mouse heart reduces heart failure mortality after myocardial infarction," Circulation, vol. 123, no. 5, pp. 504-514, 2011.

[20] D. K. Singla, X. Long, C. Glass, R. D. Singla, and B. Yan, "Induced pluripotent stem (iPS) cells repair and regenerate infarcted myocardium," Molecular Pharmaceutics, vol. 8, no. 5, pp. 1573-1581, 2011.

[21] D. K. Singla, G. E. Lyons, and T. J. Kamp, “Transplanted embryonic stem cells following mouse myocardial infarction inhibit apoptosis and cardiac remodeling," American Journal of Physiology-Heart and Circulatory Physiology, vol. 293, no. 2, pp. H1308-H1314, 2007.

[22] S. Fatma, D. E. Selby, R. D. Singla, and D. K. Singla, "Factors released from embryonic stem cells stimulate c-kit-FLK-1 ${ }^{+v e}$ progenitor cells and enhance neovascularization," Antioxidants and Redox Signaling, vol. 13, no. 12, pp. 1857-1865, 2010.

[23] C. Glass and D. K. Singla, "MicroRNA-1 transfected embryonic stem cells enhance cardiac myocyte differentiation and inhibit apoptosis by modulating the PTEN/Akt pathway in the infarcted heart," The American Journal of Physiology-Heart and Circulatory Physiology, vol. 301, no. 5, pp. H2038-H2049, 2011.

[24] C. Emanueli and P. Madeddu, "Angiogenesis gene therapy to rescue ischaemic tissues: achievements and future directions," British Journal of Pharmacology, vol. 133, no. 7, pp. 951-958, 2001.

[25] J. I. Barzilay, R. A. Kronmal, V. Bittner, E. Eaker, C. Evans, and E. D. Foster, "Coronary artery disease and coronary artery bypass grafting in diabetic patients aged $\geq 65$ years (report from the Coronary Artery Surgery Study [CASS] registry)," The American Journal of Cardiology, vol. 74, no. 4, pp. 334-339, 1994.

[26] D. Tousoulis, N. Papageorgiou, E. Androulakis et al., "Diabetes mellitus-associated vascular impairment: novel circulating biomarkers and therapeutic approaches," Journal of the American College of Cardiology, vol. 62, no. 8, pp. 667-676, 2013.

[27] A. B. Katwal and A. O. Dokun, "Peripheral arterial disease in diabetes: is there a role for genetics?" Current Diabetes Reports, vol. 11, no. 3, pp. 218-225, 2011.

[28] M. S. Ruiter, J. M. Van Golde, N. C. Schaper, C. D. Stehouwer, and M. S. Huijberts, "Diabetes impairs arteriogenesis in the peripheral circulation: review of molecular mechanisms," Clinical Science, vol. 119, no. 6, pp. 225-238, 2010.

[29] L. L. Dunn, A. M. Buckle, J. P. Cooke, and M. K. C. Ng, "The emerging role of the thioredoxin system in angiogenesis," Arteriosclerosis, Thrombosis, and Vascular Biology, vol. 30, no. 11, pp. 2089-2098, 2010.

[30] T.-L. Yue, J. Chen, W. Bao et al., "In vivo myocardial protection from ischemia/reperfusion injury by the peroxisome proliferator-activated receptor- $\gamma$ agonist rosiglitazone," Circulation, vol. 104, no. 21, pp. 2588-2594, 2001.

[31] A. A. Kocher, M. D. Schuster, M. J. Szabolcs et al., "Neovascularization of ischemic myocardium by human bone-marrowderived angioblasts prevents cardiomyocyte apoptosis, reduces remodeling and improves cardiac function," Nature Medicine, vol. 7, no. 4, pp. 430-436, 2001.

[32] G. Song, X. Li, Y. Shen et al., “Transplantation of iPSc restores cardiac function by promoting angiogenesis and ameliorating cardiac remodeling in a post-infarcted swine model," Cell Biochemistry and Biophysics, vol. 71, no. 3, pp. 1463-1473, 2015. 
[33] A. A. Qayyum, A. B. Mathiasen, and J. Kastrup, "Stem cell therapy to treat heart ischaemia: implications for diabetes cardiovascular complications," Current Diabetes Reports, vol. 14, article 554, 2014.

[34] S.-W. Kim, M. Houge, M. Brown, M. E. Davis, and Y.-S. Yoon, "Cultured human bone marrow-derived $\mathrm{CD} 31^{+}$cells are effective for cardiac and vascular repair through enhanced angiogenic, adhesion, and anti-inflammatory effects," Journal of the American College of Cardiology, vol. 64, no. 16, pp. 1681-1694, 2014.

[35] M. Clarke, M. Bennett, and T. Littlewood, "Cell death in the cardiovascular system," Heart, vol. 93, no. 6, pp. 659-664, 2007.

[36] J.-Y. Huang and J.-I. Chuang, "Fibroblast growth factor 9 upregulates heme oxygenase- 1 and $\gamma$-glutamylcysteine synthetase expression to protect neurons from 1-methyl-4-phenylpyridinium toxicity," Free Radical Biology and Medicine, vol. 49, no. 6, pp. 1099-1108, 2010.

[37] L. DiNapoli, J. Batchvarov, and B. Capel, "FGF9 promotes survival of germ cells in the fetal testis," Development, vol. 133, no. 8, pp. 1519-1527, 2006.

[38] L. Ye, P. Zhang, S. Duval, L. Su, Q. Xiong, and J. Zhang, “Thymosin $\beta 4$ increases the potency of transplanted mesenchymal stem cells for myocardial repair," Circulation, vol. 128, no. 1, pp. S32-S41, 2013.

[39] Y.-Y. He, Y. Wen, X.-X. Zheng, and X.-J. Jiang, "Intramyocardial delivery of HMGB1 by a novel thermosensitive hydrogel attenuates cardiac remodeling and improves cardiac function after myocardial infarction," Journal of Cardiovascular Pharmacology, vol. 61, no. 4, pp. 283-290, 2013.

[40] D. Tempel, M. de Boer, E. D. van Deel et al., "Apelin enhances cardiac neovascularization after myocardial infarction by recruiting aplnr+ circulating cells," Circulation Research, vol.111, no. 5, pp. 585-598, 2012.

[41] X. Hu, R. Wu, Z. Jiang et al., "Leptin signaling is required for augmented therapeutic properties of mesenchymal stem cells conferred by hypoxia preconditioning," Stem Cells, vol. 32, no. 10, pp. 2702-2713, 2014. 


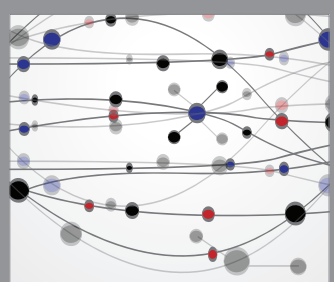

The Scientific World Journal
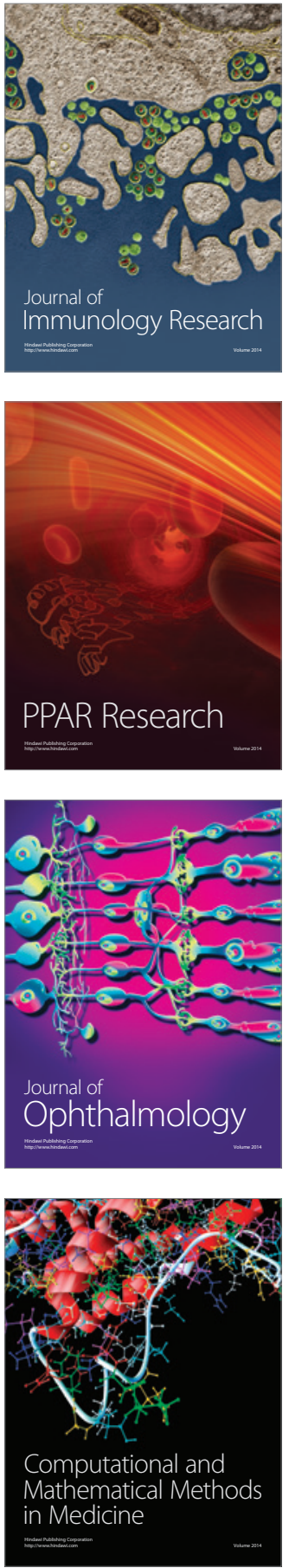

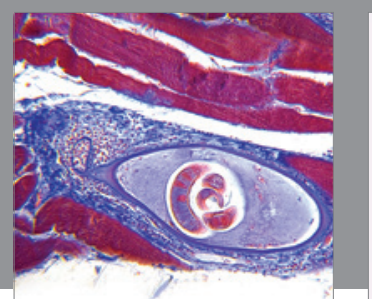

Gastroenterology Research and Practice

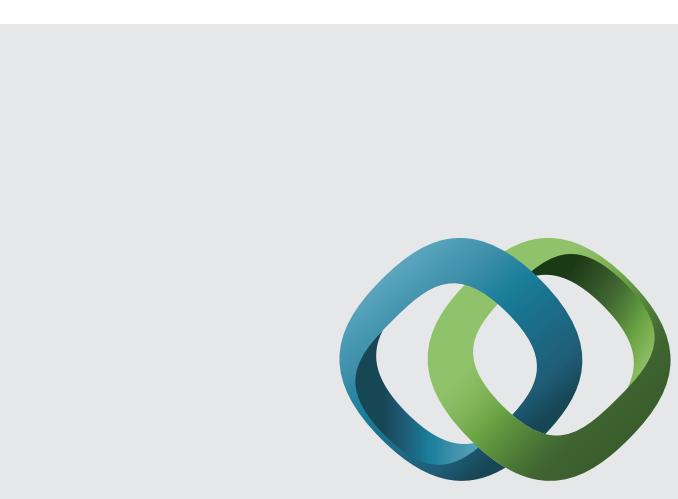

\section{Hindawi}

Submit your manuscripts at

http://www.hindawi.com
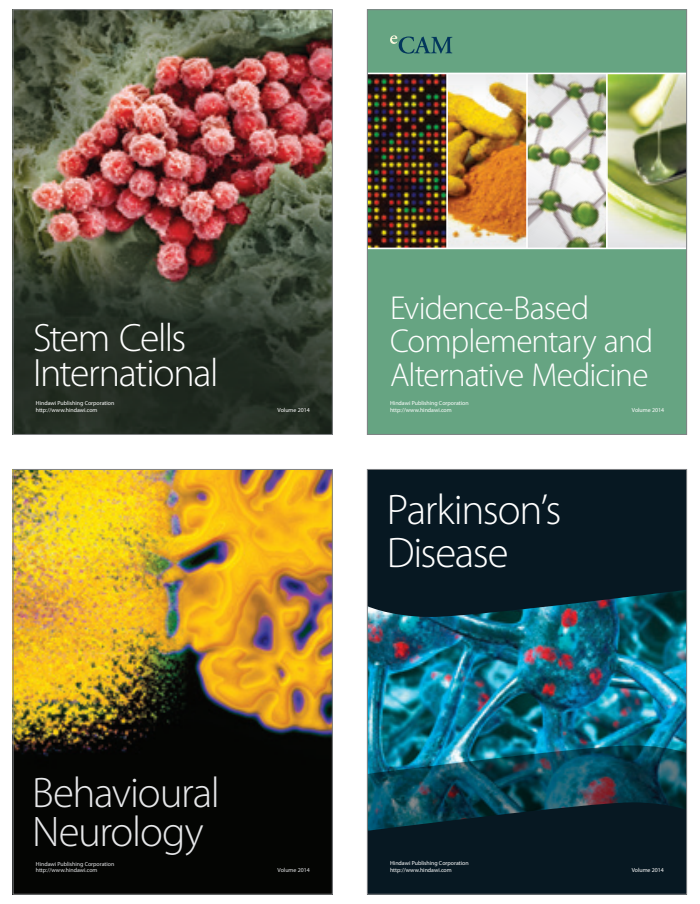
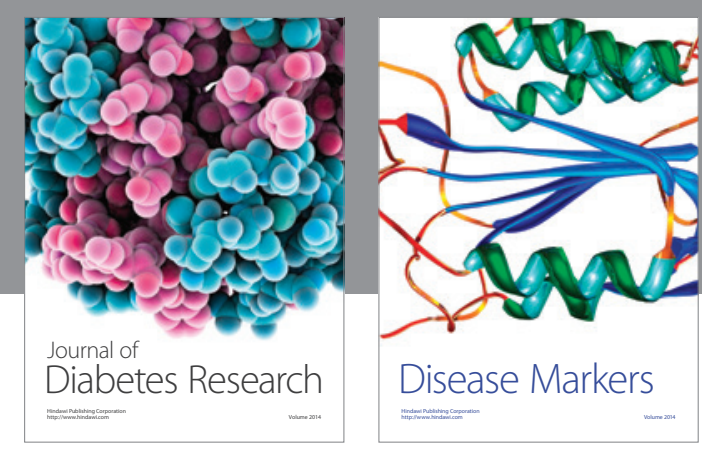

Disease Markers
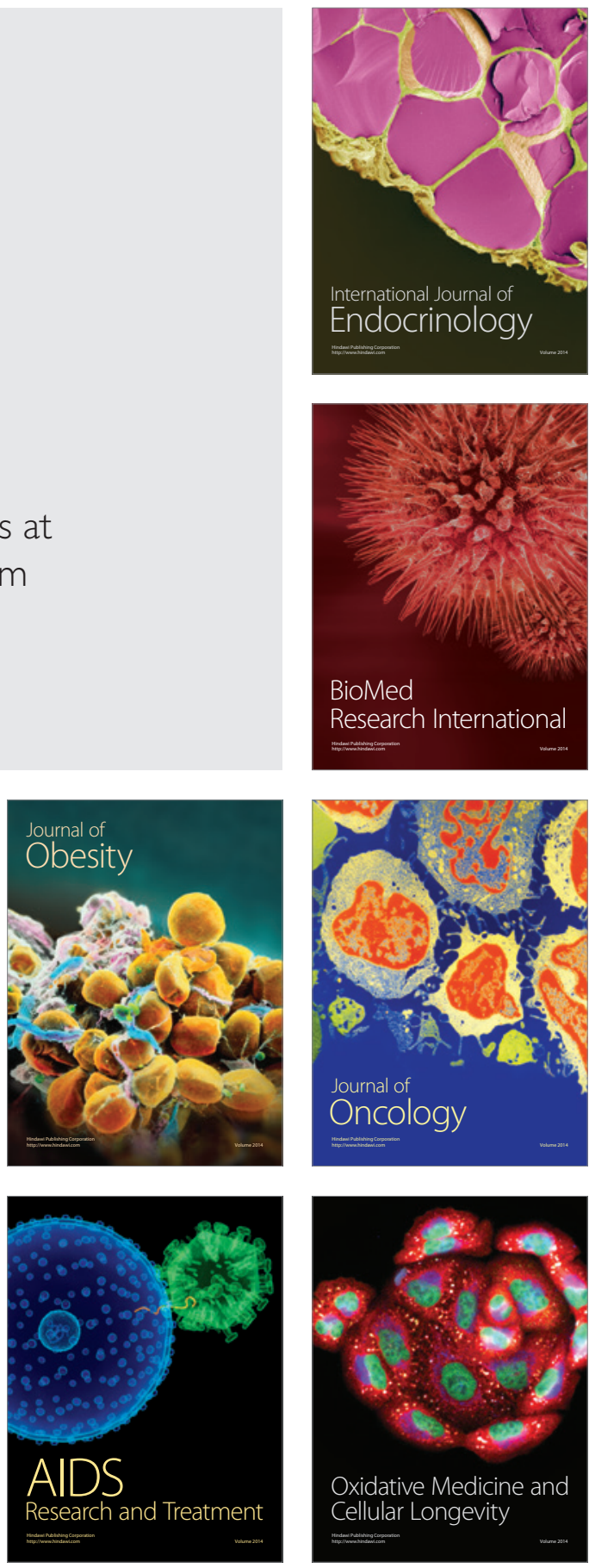